\title{
VENA FEMORAL BITRONCULAR CON TRONCO AXIOFEMORAL ORIGINADO DE LA VENA SAFENA PARVA
}

\section{FEMORAL VEIN BITRONCULAR ORIGINATED WITH THE TRUNK AXIOFEMORAL PARVA SAPHENOUS VEIN}

\author{
Ricardo Luque ${ }^{1}$, Yobany Quijano ${ }^{2}$, Edison Peralta ${ }^{3}$, Angy Villamil $^{4}$, Manuel Rojas ${ }^{5}$, Johanna Diaz ${ }^{6}$
}

\begin{abstract}
1 Médico Cirujano, docente Departamento de Morfología Humana, Facultad de Medicina, Universidad de Ciencias Aplicadas y Ambientales U.D.C.A, e-mail: riluque@udca.edu.co; 2 Médico Cirujano, Profesor titular Departamento de Morfología Humana, Facultad de Medicina, Universidad de Ciencias Aplicadas y Ambientales U.D.C.A, autor para correspondencia: email: globdimorf@udca.edu.co; 3 Médico Cirujano, docente Departamento de Morfología Humana, Facultad de Medicina, Universidad de Ciencias Aplicadas y Ambientales U.D.C.A, e-mail: eperalta@udca.edu.co; 4 Médica docente, Departamento de Morfología Humana, Facultad de Medicina, Universidad de Ciencias Aplicadas y Ambientales U.D.C.A, e-mail: anvillamil@udca.edu.co; 5 Estudiante de Medicina Humana, Universidad de Ciencias Aplicadas y Ambientales U.D.C.A, e-mail: manuel_9316@udca.edu.co; 6 Estudiante de Medicina Humana, Universidad de Ciencias Aplicadas y Ambientales U.D.C.A, e-mail: jodiaz@udca.edu.co. Bogotá-Colombia, calle 222 No. 54-37
\end{abstract}

Rev. U.D.C.A Act. \& Div. Cient. 17(2): 325-331, Julio-Diciembre, 2014

\section{RESUMEN}

El conocimiento de la anatomía de las venas de los miembros inferiores del humano es fundamental para comprender la fisiopatología, el diagnóstico y el tratamiento de patologías vasculares. La mayoría de errores en el diagnóstico y en el tratamiento de estas patologías obedece a variaciones anatómicas. En este estudio, se reflexiona, se analiza y se discute sobre las implicaciones que en la clínica y en las nuevas investigaciones en morfología tiene una variación anatómica, encontrada en un cadáver de género femenino, en el cual, se evidenció una duplicación de la vena poplítea, de una vena que pasa a través del hiato aductor, mientras que la otra asciende por la cara posterior del muslo, siguiendo el trayecto del nervio ciático. A pesar que las venas de los miembros inferiores guardan algunas características comunes, cada vez se describe -con mayor detalle- un elevado número de variaciones, lo que influye en la búsqueda de trombos descritos en artículos de revistas indexadas y no se tienen en cuenta en otros patrones de distribución.

Palabras clave: Variaciones anatómicas, vena poplítea, género femenino, duplicación, várices miembros inferiores.

\section{SUMMARY}

Knowledge of the anatomy of the veins of the lower limbs is fundamental to understanding the pathophysiology, diagnosis and treatment of vascular diseases. Most errors in the diagnosis and treatment of these diseases is due to anatomical variations thereof. This study analyzes and anatomical variation found in a female body, in which a duplication of the popliteal vein, vein one passing through the adductor hiatus evidenced discussed, while the other ascends the face posterior of the thigh along the path of the sciatic nerve. While the veins of the lower limbs stored common characteristics, each time is described in greater detail a large number of variations which influences thrombus search items described in refereed journals and are not taken into account other patterns distribution.

Key words: Anatomical variations, popliteal vein, female, duplication, varicose legs.

\section{INTRODUCCIÓN}

El sistema venoso de la extremidad inferior de un humano, se divide en un sistema venoso profundo, que viaja por la extremidad, paralelo a las arterias y un sistema venoso superficial, de localización subcutánea, que drena en el sistema venoso profundo.

El sistema venoso profundo inicia en el pie, con dos sistemas en paralelo; el primero, conocido como sistema dorsal y anterior, a través del arco venoso plantar profundo y la vena metatarsal profunda, las cuales, van a drenar a las dos venas tibiales anteriores. El segundo, dominado como sistema plantar y posterior, formado por las venas digitales plantares y las metatarsales plantares, que van a formar las venas tibiales posteriores (Latarjet \& Ruiz Liard, 2004; Cag- 
giati et al. 2002). En la pierna, las venas tibiales anteriores, las tibiales posteriores y las fibulares, ascienden y convergen posteriormente, a nivel del arco tendinoso del sóleo, para formar la vena poplítea que, en aproximadamente el $8 \%$ de las extremidades, se presenta un verdadero sistema venoso poplíteo duplicado (Bannister et al. 2001; Sadowska et al. 2013). Dicha vena, atraviesa el canal aductor para ingresar al compartimiento anterior del muslo, como vena femoral, que discurre por el canal femoral (Hunter) hasta encontrarse con la vena femoral profunda, a nivel del triángulo femoral o de Scarpa, donde forman la vena femoral común (Giraldo, 2005; Caggiati et al. 2005); esta vena cuando cruza el ligamento inguinal en sentido ascendente, se denomina vena iliaca externa.

El sistema venoso superficial tiene un recorrido subcutáneo y sus dos principales exponentes son la vena safena magna, que recorre la cara medial de la pierna y el muslo. Cuando llega al tercio proximal, este último realiza un arco, que le permite desembocar en la vena femoral común, a nivel del triángulo femoral (Latarjet \& Ruiz Liard, 2004). La segunda vena del componente venoso superficial es la vena safena parva, que recorre la cara posterior del muslo hasta drenar, en aproximadamente el $70 \%$ de los casos, en la vena poplítea $y$, en los restantes, en la vena femoral, en el $25 \%$ y, en la vena safena magna, en el 5\% restante (Sandoval et al. 2013).

Embriológicamente, las venas se desarrollan a partir de la inducción ectodérmica de los nervios en crecimiento sobre el mesodermo extraembrionario, lo que genera el crecimiento de los vasos sanguíneos que se van a extender, siguiendo el trayecto de los nervios que inducen su formación (Mukouyama et al. 2002). En las extremidades inferiores, el desarrollo de los vasos sanguíneos se produce siguiendo el trayecto de los tres grandes nervios que ingresan al muslo, el nervio femoral anteriormente y el nervio ciático en la región posterior profunda y el nervio cutáneo femoral posterior, ubicado en la región posterior superficial. La inducción del nervio genera el desarrollo de tres grandes grupos de vasos sanguíneos. El primer grupo, se produce por el estímulo del nervio femoral, que genera los vasos femorales y son la arteria y la vena femorales, que se conocen como vasos preaxiales, por su relación con el nervio ciático (Parry et al. 2002). En un segundo grupo están los vasos sanguíneos, que siguen el trayecto del nervio ciático y están la arteria y la vena ciática, conocidos como vasos axiales. Estos son los principales responsables de la irrigación y en drenaje venoso del miembro inferior en esta etapa embrionaria, pero involucionan hasta desaparecer en la etapa embrionaria tardía (Cardoso et al. 2010; Uhl et al. 2010). El tercer tipo, se denomina patrón postaxial, que corresponde a un drenaje venoso y circulación arterial, que sigue el trayecto del nervio cutáneo femoral posterior y, por tanto, se ubica posterior al trayecto del nervio ciático, denominándose postaxial (Mukouyama et al. 2002; Uhl et al. 2010).
Las enfermedades vasculares venosas de los miembros inferiores son condiciones crónicas con una alta prevalencia, que incluyen la insuficiencia venosa y la enfermedad varicosa (Beebe-Dimmer et al. 2005). Son patologías con una alta prevalencia en la población general y con importantes consecuencias, como trombosis venosa, con el consiguiente riesgo de tromboembolismo pulmonar, las dermatitis y úlceras varicosas secundarias, que afectan la calidad de vida de los pacientes y sus familiares; algunas estudios calculan la incidencia general de insuficiencia venosa crónica en el $1 \%$, de la población adulta y el $4 \%$, de la población mayor de 65 años (Word, 2010). Las enfermedades más comunes incluyen las várices de miembros inferiores, la insuficiencia arterial y la trombosis venosa profunda, entre las más frecuentes. Dichas patologías presentan una alta prevalencia en la población general, siendo las várices muy frecuentes entre mujeres adultas y la insuficiencia arterial, en personas ancianas. La trombosis venosa profunda, aunque no tiene una alta incidencia, tiene unas complicaciones tan serias que pueden afectar la funcionalidad de los pulmones e, incluso, comprometer la vida (Eklof et al. 2009).

Las várices de miembros inferiores tienen una alta prevalencia en la población, reportando, en algunas publicaciones, que pueden afectar entre un 20 y $65 \%$ de los trabajadores de la salud en Chile, donde el $75 \%$ es en mujeres y $25 \%$ en los hombres, de los cuales, un $76 \%$ presenta dolor y un 33\% edema en los miembros inferiores (Espínola et al. 2007). Otros estudios, en países de habla inglesa, refieren una prevalencia con amplias fluctuaciones, explicadas por las características propias del estudio, que pueden ir de un 2 a un 56\%, en hombres y, del 1 al 73\%, en mujeres (BeebeDimmer et al. 2005). Algunos factores de riesgo asociados a la aparición de várices de miembros inferiores son la obesidad, género femenino, historia familiar, antecedentes de trombosis venosa profunda y edad avanzada (Kouri, 2009; Ouahes \& Phillips, 1995).

Las várices de miembros inferiores, se caracterizan clínicamente por síntomas difusos, como dolor en las extremidades, prurito y "fatiga de piernas". El examen físico evidencia dilataciones en trayectos venosos, edema o signos de reflujo venoso (Kouri, 2009). La enfermedad varicosa de miembros inferiores, aparte de los síntomas propios de la enfermedad, presenta diversas complicaciones asociadas, afectando de una forma importante la salud y la calidad de vida de las personas que la padecen (Kanchanabat et al. 2010).

Las ulceras de miembros inferiores, asociadas a varices, tienen una prevalencia que fluctúa entre el 0,1 y el $1 \%$ de la población, de acuerdo a diferentes estudios (González, 2003) y se caracterizan por ubicarse, por lo general, a nivel maleolar, presentar bordes irregulares, estar rodeadas de zonas de hemosiderosis y tener tendencia a la cronicidad, que puede 
variar de algunos meses a muchos años (Ouahes \& Phillips, 1995). Dado su curso insidioso, el costo tanto económico como en calidad de vida para el sistema de salud, la sociedad y el paciente mismo, son muy altos.

Por otro lado, la trombosis venosa profunda puede llegar a afectar a dos personas de cada mil (Páramo et al. 2007), una incidencia muy alta, dado el carácter catastrófico por las posibles consecuencias, entre las que se encuentra, el síndrome post-trombótico y el tromboembolismo pulmonar (TVP), éste último, con altas tasas de mortalidad (Quinlan et al. 2003). El diagnóstico de la trombosis venosa profunda depende de una adecuada sospecha clínica, detallando los antecedentes personales, la enfermedad actual y un buen examen físico y, ante la sospecha de trombosis venosa profunda, se debe realizar su confirmación, a partir de exámenes paraclínicos e imágenes diagnósticas, específicamente, el ultrasonido que, al no ser invasivo y ser más específico que la flebografía y los estudios de química sanguínea, resulta ser el método diagnóstico de elección (Merli, 2008). El ultrasonido es un método altamente sensible y específico para el diagnóstico de trombosis venosa profunda, alcanzando una sensibilidad y especificidad, que fluctúan entre 95 y 96\%; sin embargo, el alto porcentaje reportado de falsos negativos, en la mayoría de los casos, son producidos por variaciones anatómicas de las venas de miembros inferiores, en especial, la duplicación de la vena femoral (Hamper et al. 2007).

Las patologías venosas de miembros inferiores son una causa importante de morbimortalidad en la población general. Su diagnóstico es a través de imágenes diagnósticas y su tratamiento es principalmente quirúrgico. Esto implica que es fundamental un conocimiento amplio de la anatomía de los componentes venosos, tanto superficial como profundo de los miembros inferiores, lo que incluye las variaciones anatómicas, que puede presentar dicho sistema, dado que una gran parte de los errores cometidos durante la ejecución e interpretación de los procedimientos diagnósticos no invasivos, como el ultrasonido y métodos invasivos, como flebografías, son producidos por dichas anomalías anatómicas, por lo cual, es importante describir variantes anatómicas nuevas o poco frecuentes de las venas de los miembros inferiores, para proporcionar más herramientas, que permitan optimizar los procesos diagnósticos y terapéuticos y afianzar, de esta forma, una práctica médica más segura y eficaz (Park et al. 2011).

Este trabajo reflexiona, a través de la presentación de un caso clínico, sobre la importancia de tener presentes las variaciones anatómicas en la interpretación de las imágenes diagnósticas de los miembros inferiores y en el abordaje quirúrgico para el tratamiento de las patologías venosas de esta región.

\section{MATERIALES Y MÉTODOS}

Dentro de los cadáveres que se encuentran en el anfiteatro del Programa de Medicina de la U.D.C.A, de Bogotá, Colombia, donados como parte del convenio con el Instituto de Medicina Legal y Ciencias Forenses. En un cadáver de género femenino, fijado en formaldehido al $10 \%$, nacionalidad colombiana, se realizó una disección de miembros inferiores, de la siguiente manera: 1) Se colocó el cadáver en decúbito dorsal y, 2) Se disecó por planos la región glútea, la cara posterior del muslo y la pierna y, luego, en posición en decúbito ventral, se procedió a disecar la cara anterior del muslo.

El cadáver correspondía a una mujer joven, sin malformaciones congénitas visibles, ni trastornos del desarrollo de las extremidades, evidentes al examen. Tampoco se observaron otras variaciones anatómicas relevantes en la extremidad inferior, ni en otro sitio del cuerpo.

Para la realización de este artículo, se siguieron todos los lineamientos del decreto 8430 de 1993, le declaración de Helsinki y el código de Núremberg, contando, por lo tanto, con la autorización del Comité de Ética institucional.

\section{RESULTADOS Y DISCUSIÓN}

Se realizó examen detallado de la anatomía del sistema venoso profundo, lo que permitió evidenciar la presencia de la vena tibial posterior, las vena tibial anterior y la fibular, emergiendo a nivel del arco tendinoso del sóleo y convergiendo para formar la vena poplítea, la cual, tenía como particularidad, que no evidenciaba la llegada de la vena safena externa. La vena poplítea ascendía por la fosa poplítea y se dirigía anteriormente, hasta atravesar el hiato aductor. Posteriormente, dicha vena continuaba como vena femoral por el canal femoral de Hunter y, luego, se dirigía al triangulo femoral, donde se encuentra con la vena femoral, para forman la vena femoral (Fotografía 1).

Se evidencia, adicionalmente, cómo a nivel de la fosa poplítea la vena safena parva recibe una "tributaria" de la vena tibial posterior y forma un segundo sistema venoso profundo que, en lugar de dirigirse hacia el hiato aductor, continúa por el compartimiento posterior del muslo, adyacente al nervio ciático, ascendiendo entre los músculos bíceps femoral, semitendinoso y semimembranoso posteriormente y el músculo aductor mayor anteriormente (Fotografía 1). Al llegar al tercio superior del muslo, dicha vena penetra a través de un hiato formado por la inserción proximal en el fémur del músculo aductor mayor, por donde penetra al compartimento anterior del muslo, para drenar en la vena femo ral profunda, justo antes de que ésta se encuentre con la vena femoral (Fotografía 2). 


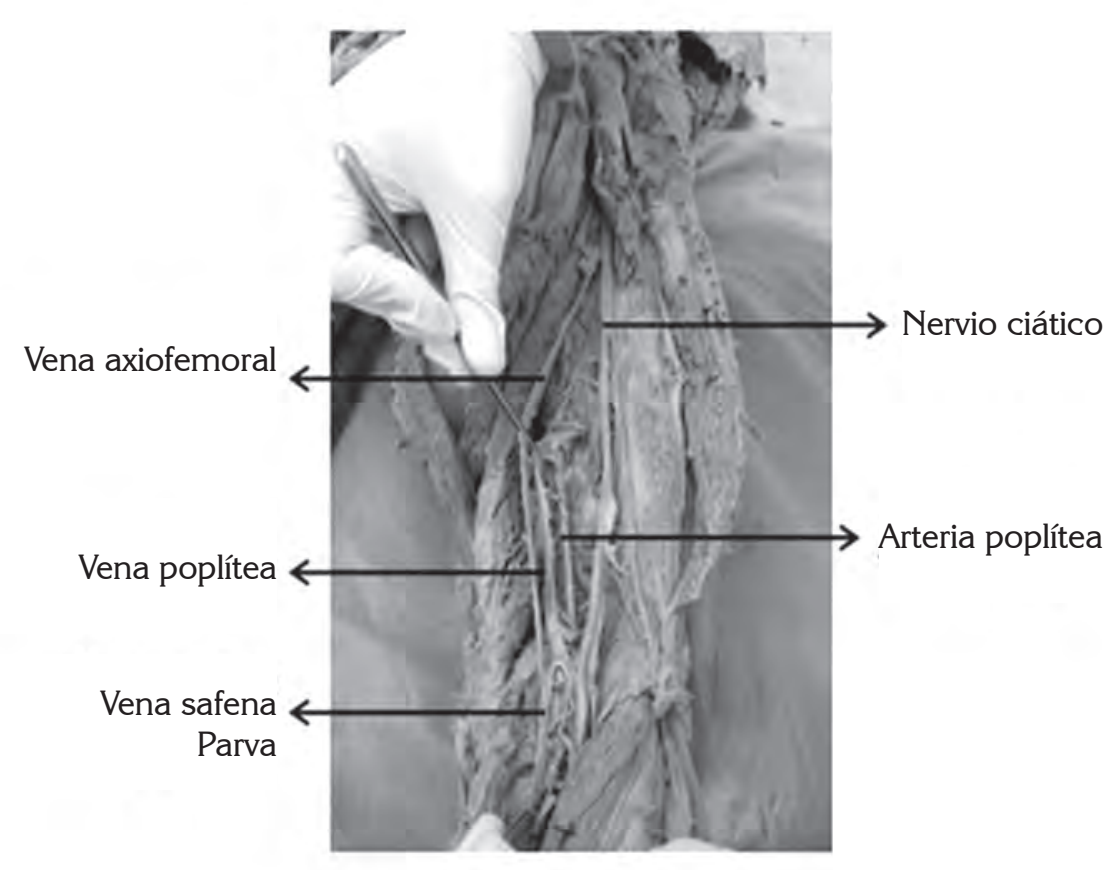

Fotografía 1. Cara posterior del muslo, que evidencia vena axiofemoral emergiendo de la vena safena externa y vena poplítea, conectándose con ella por un pequeño vaso comunicante.

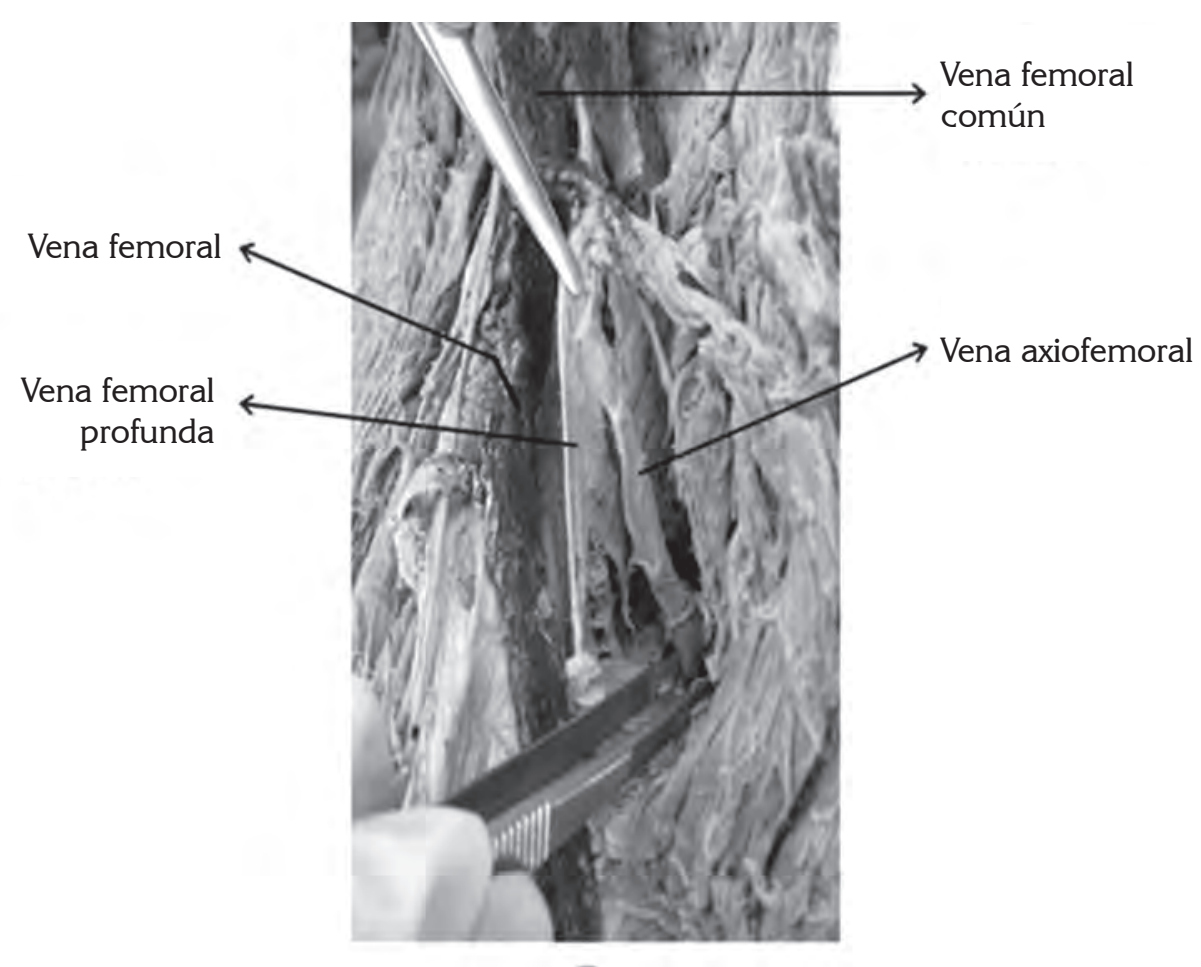

Fotografía 2. Cara anterior y proximal del muslo, que muestra cómo la vena axiofemoral pasa a través de un canal formado por la inserción proximal del músculo aductor mayor y cómo desemboca posteriormente en la vena femoral profunda. 
Como se evidencia por la descripción y se confirma en las fotografías, al observar el sistema venoso accesorio descrito, la configuración bitroncular corresponde a una bifurcación baja; uno de los troncos corresponde a la vena femoral superficial y, el otro tronco, denominado axiofemoral, por seguir el trayecto del nervio ciático, que va a desembocar en la vena femoral profunda para terminar encontrándose con la vena femoral superficial y formar la vena femoral. De acuerdo a los reportes previos de prevalencia de malformaciones venosas y de variaciones anatómicas de miembros inferiores, esta variación anatómica tiene una frecuencia del $5 \%$ de la población, en el único estudio encontrado, que reporta dicho tipo de variaciones (Uhl et al. 2010). Otros estudios, por lo general, exploran variaciones en la vena femoral propiamente y en el compartimento anterior del muslo, en gran parte, debido a que son investigaciones realizadas por imágenes diagnósticas, que no registran dichas variaciones, ya sea por imposibilidad técnica o porque no es el objetivo mismo del estudio. Por otro lado, se considera poco probable que dicha vena corresponda a la vena ciática, ya que desemboca en las venas glúteas y la vena axiofemoral, descrita aquí, lo hace a nivel de la vena femoral y sólo sigue el trayecto del nervio ciático en los tercios distal y medio de la cara posterior del muslo, contrario a lo descrito para la vena ciática, que puede tener un inicio alto, como tercio medio del muslo, pero siempre acompaña al ciático hasta la región glútea (Cardoso et al. 2010)

Quinlan et al. (2003), en un estudio retrospectivo utilizando venografías tomadas a 404 pacientes, como parte de una investigación de profilaxis de trombosis, encontraron que entre el 70 y el $75 \%$ de las personas evaluadas tienen las venas de las piernas en duplicado, mientras el restante 25 a 30\%, los tienen en disposición clásica. Cerca del $55 \%$ de los individuos presentan una única vena poplítea, mientras el restante $45 \%$, muestra dos o más venas poplíteas (Tabla 1). En ese estudio, se reportan venas femorales duplicadas, pero todas en la región anterior del muslo, por lo general, iniciado en el tercio medio del muslo (Quinlan et al. 2003).

Tabla 1. Tabla comparativa del recorrido de las venas femoral y poplítea, según estudios.

\begin{tabular}{|c|c|c|c|c|c|c|c|c|c|c|}
\hline ESTUDIO & POBLACIÓN & VASO & MMIIss. & DISPOSICIÓN & DER & IZQ & TB & T.A & V.F.P & Total \\
\hline \multirow{2}{*}{ Uhl et al. 2010} & $168(336)$ & \multirow{7}{*}{ V.F } & 306 & Unitroncular & & & & & & $91 \%$ \\
\hline & & & 30 & $\begin{array}{l}\text { Duplicación } \\
\text { bitroncular }\end{array}$ & & & $2 \%$ & $4,8 \%$ & $2 \%$ & $9 \%$ \\
\hline \multirow{5}{*}{ Casella et al. 2010} & \multirow[b]{3}{*}{$157(314)$} & & 141 & Sin Duplicación & $26,8 \%$ & $17 \%$ & & & & $43,8 \%$ \\
\hline & & & 82 & $\begin{array}{l}\text { Duplicación } \\
\text { Completa }\end{array}$ & $8 \%$ & $18 \%$ & & & & $26 \%$ \\
\hline & & & 80 & Duplicación Distal & $18 \%$ & $7 \%$ & & & & $25 \%$ \\
\hline & & & 9 & $\begin{array}{l}\text { Duplicación } \\
\text { Proximal }\end{array}$ & $0,9 \%$ & $1,9 \%$ & & & & $2,8 \%$ \\
\hline & & & 2 & Otros Patrones & & & & & & $0,6 \%$ \\
\hline \multirow{3}{*}{ Quinlan et al. 2003} & & \multirow{3}{*}{ V.P } & 452 & Única & 222 & 230 & & & & $55,9 \%$ \\
\hline & & & 337 & Doble & 172 & 165 & & & & $41,7 \%$ \\
\hline & 404(808) & & 19 & Triple & 10 & 9 & & & & $2,4 \%$ \\
\hline
\end{tabular}

T.B: Tronco bífido, T.A: Tronco axial, V.F.P: Vena femoral profunda, V.F: Vena femoral, V.P: Vena Poplítea., MMIlss.: Miembros inferiores, (-)Total de miembros inferiores examinados. 
En un estudio realizado a 157 individuos, a quienes les visualizaron ambas piernas, a través de dúplex scan, Benaduce et al. (2010) encontraron que el 55\% de los pacientes presentaron alguna duplicación de la vena femoral, describiendo tres tipos de duplicaciones característicos. Al primer tipo, lo denominaron duplicación completa tipo I, donde las dos venas femorales se extienden desde la vena poplítea a la vena femoral o vena femoral común; el segundo tipo, lo identificaron duplicación distal tipo II, cuando la vena femoral accesoria drena en la parte media del muslo y el tercer tipo, lo llamaron duplicación proximal tipo III, que se presenta cuando la vena femoral accesoria drena a nivel de la vena femoral adyacente a la desembocadura de la vena femoral profunda (Tabla 1).

Uno de los trabajos más completos que se ha realizado (Uhl et al. 2010), en el que se analizaron 336 miembros inferiores de cadáveres frescos, a los que se les realizaron lavado de venas e inyección con látex. Ellos clasificaron el desarrollo de las venas en tres grupos, de acuerdo al eje de desarrollo vascular de la extremidad, que es el nervio ciático, dado que el crecimiento vascular se deriva de la inducción que realizan los nervios primitivos sobre los vasos sanguíneos (Mukouyama et al. 2002). Así, los que se ubican delante de este, se denominan preaxiales y su eje de desarrollo es el nervio femoral; los que se ubican a nivel de éste, se denominan axiales y los que se hallan posterior, se llaman postaxiales y su inductor del desarrollo será el nervio cutáneo femoral posterior o nervio ciático menor (Uhl et al. 2010). En dicho estudio, los autores encontraron que el $88 \%$ de las configuraciones vasculares corresponden a la configuración venosa clásica, mientras el $12 \%$ restante concierne a variaciones anatómicas, que denominan malformaciones vasculares congénitas. De acuerdo a la disposición de los vasos sanguíneos de la extremidad y al flujo que soportan, clasificaron a las venas en dos grupos: uno, denominado unitroncular, donde una vena carga con una gran parte del flujo sanguíneo venoso de la extremidad y el otro, bitroncular, donde dos venas son responsables, de manera más o menos similar, del flujo sanguíneo. El $91 \%$ de las extremidades evaluadas tenían una disposición unitroncular, de las cuales, un $88 \%$, con disposición clásica y un $3 \%$, correspondiente a las variaciones anatómicas; el 9\% restante presentó una disposición bitroncular, que se pueden, a su vez, dividir en cuatro grupos. El primero, es una forma bífida, donde las dos venas ascienden paralelas por la fosa poplítea y entran al muslo por el canal aductor, donde terminan fusionándose; el segundo grupo, se denomina plexiforme, ya que las dos venas ascienden en paralelo, pero presentan múltiples vasos comunicantes, lo que les da un aspecto de red; el tercer tipo, es una bifurcación alta, donde las dos venas se bifurcan en el muslo y, posteriormente, vuelven a converger en el muslo proximal y el cuarto tipo, corresponde a una bifurcación baja, donde las dos venas emergen en la fosa poplítea y una asciende siguiendo el trayecto habitual de la vena femoral superficial, mientras la otra asciende siguiendo el trayecto del nervio ciático, denominado tronco axiofemoral (Tabla 1).

Las variaciones anatómicas de las venas de los miembros inferiores son frecuentes y pueden ser un factor de confusión en el diagnóstico de patologías tan relevantes, como la trombosis venosa profunda, ya que los trombos solamente se buscan en el trayecto clásico, descrito en la literatura y no se tienen en cuenta otros patrones de distribución.

Conflicto de intereses: El manuscrito fue preparado y revisado con la participación de todos los autores, quienes declaramos que no existe conflicto de intereses, que ponga en riesgo la validez de los resultados presentados.

\section{BIBLIOGRAFÍA}

1. BANNISTER, L.; BERRY, M.; COLLINS, P.; DYSON, M.; DUSSEK, J.; FERGUSON, M. 2001. Anatomía de Gray. Ed. Harcourt. Madrid. Edición. 38:530-553.

2. BEEBE-DIMMER, J.; PFEIFER, J.R.; ENGLE, J.S.; SCHOTTENFELD, D. 2005. The epidemiology of chronic venous insufficiency and varicose veins. Ann. Epidemiol. 15(3):175-184.

3. BENADUCE, I.; PRESTI, C.; YAMAZAKI, Y.; VASSOLER, A.; FURUYA, L.; SABBAG, C. 2010. A duplex scanbased morphologic study of the femoral vein: Incidence and patterns of duplication. Vascular Med. 15(3):197-203.

4. CAGGIATI, A.; BERGAN, J.J.; GLOVICZKI, P.; JANTET, G.; WENDELL-SMITH, C.P.; PARTSCH, H. 2002. Nomenclature of the veins of the lower limbs: An international interdisciplinary consensus statement. J. Vasc. Surgery. 36(2):416-422.

5. CAGGIATI, A., BERGAN, J.J., GLOVICZKI, P., EKLOF, B., ALLEGRA, C.; PARTSCH, H. 2005. Nomenclature of the veins of the lower limb: extensions, refinements, and clinical application. J. Vasc. Surgery. 41(4):719724.

6. CARDOSO, B.B.; ALVARENGA, C.O.; MIYAHARA, M.D.S.; BURIHAN, M.C.; LIMA, M.R.Q.A.D.; KUWAHARA, M.C.; SILVA, R.C.M. 2010. Persistent sciatic vein. J. Vasc. Bras. 9(3):137-140.

7. CASELLA, I.B.; PRESTI, C.; YAMAZAKI, Y.; VASSOLER, A.A.; FURUYA, L.A.; SABBAG, C.D. 2010. A duplex scan-based morphologic study of the femoral vein: incidence and patterns of duplication. Hematology 
Week. News RX. High Beam Research. Disponible desde Internet en: http://www.highbeam.com> (con acceso el 11/05/2013).

8. EKLOF, B.; PERRIN, M.; DELIS, K.T.; RUTHERFORD, R.B.; GLOVICZKI, P. 2009. Updated terminology of chronic venous disorders: the VEIN-TERM transatlantic interdisciplinary consensus document. J. Vasc. Surgery. 49(2):498-501.

9. ESPÍNOLA, C.F.; BERNAL, M.; AUCEJO, M.; VILLALBA, J.C. 2007. Prevalencia de várices en miembros inferiores en el personal del Hospital de Clínicas. Rev. Chilena Cirugía. 59(5):342-347.

10. GIRALDO, O. 2005. Nomenclatura actual de la anatomía venosa de los miembros inferiores y correlación ecográfica. Rev. Col. Cirugía Vasc. 5(1):11-17.

11. GONZÁLEZ-GÓMEZ, A. 2003. Prevención y calidad de vida en pacientes con úlceras vasculares. Angiologia. 55(3):280-284.

12. HAMPER, U.; DEJONG, M.R. 2007. Ultrasound evaluation of the lower extremity veins. Radiol. Clin. N. Am. 45:525-547.

13. KANCHANABAT, B.; WONGMAHISORN, Y.; STAPANAVATR, W.; KANCHANASUTTIRAK, P.; MANOMAIPHIBOON, A. 2010. Clinical presentation and patterns of venous reflux in Thai patients with chronic venous insufficiency (CVI). Eur. J. Vasc. Endovasc. Surgery. 40(3):399-402.

14. KOURI, B. 2009. Current evaluation and treatment of lower extremity varicose veins. Am. J. Med. 122(6):513 -515.

15. LATARJET, M.; RUIZ LIARD, A. 2004. Anatomía humana. $4^{\mathrm{a}}$ ed. Buenos Aires. p.836-841.

16. MERLI GENO, J. 2008. Pathophysiology of venous thrombosis and the diagnosis of deep vein thrombosis-pulmonary embolism in the elderly. Cardiol. Clin. 26:203-219.

17. MUKOUYAMA, Y.S.; SHIN, D.; BRITSCH, S; TANIGUCHI, M.; ANDERSON, D.J. 2002. Sensory nerves determine the pattern of arterial differentiation and blood vessels branching in the skin. Cell. 109:693-705.
18. OUAHES, N.; PHILLIPS, T.J. 1995. Leg ulceras. Current Problems Dermatol. 7(4):114-142.

19. PÁRAMO, J.A.; RUIZ DE GAONA, R.; GARCÍA, P.; RODRÍGUEZ, R. 2007. Diagnóstico y tratamiento de la trombosis venosa profunda. Rev. Med. Univ. Navarra. 51(1):13-17.

20. PARK, E.; WOOK CHUNG, J.; LEE, W.; YIN, Y.H.; HA, J.; KIM, S.J.; PARK, J. 2011. Three-dimensional evaluation of the anatomic variations of the femoral vein and popliteal vein in relation to the accompanying artery by using ct venography. Korean J. Radiol 12(3):327-340.

21. PARRY, D.J.; ALDOORI, M.I.; HAMMOND, R.J.; KESSEL, D.O.; WESTON, M.; SCOTT, D.J. 2002. Persistent sciatic vessels, varicose veins, and lower limb hypertrophy: An unusual case or discrete clinical syndrome? J. Vasc. Surgery. 36(2):396-400.

22. QUINLAN, D.; ALIKHAN, R.; GISHEN, P.; SIDHU, P. 2003. Variations in lower limb venous anatomy: implications for US diagnosis of deep vein thrombosis. Radiology. 228(2):443-448.

23. SADOWSKA, A., SPODNIK, J.H.; WÓJCIK, S. 2013. Variations in popliteal fossa venous anatomy: implications for diagnosis of deep-vein thrombosis. Folia Morphol. 72:51-56.

24. SANDOVAL MARTÍNEZ, C.A.; VARGAS CUENCA, C.; GONZÁLEZ BRAVO, O. 2013. Prevalencia de las variaciones anatómicas de la desembocadura de la vena safena menor en pacientes del servicio de urgencias del Hospital Militar Central del 2006 al 2008.

25. UHL, J.F.; GILLOT, C.; CHAHIM, M. 2010. Anatomical variations of the femoral vein. J. Vasc. Surgery. 52(3):714-719.

26. WORD, R. 2010. Medical and surgical therapy for advanced chronic venous insufficiency. Surg. Clin. North Am. 90(6):1195-1214.

Recibido: Marzo 14 de 2014

Aceptado: Octubre 10 de 2014

\section{Como citar:}

Luque, R.; Quijano, Y.; Peralta, E.; Villamil, A.; Rojas, M.; Díaz, J. 2014. Vena femoral bitroncular con tronco axiofemoral originado de la vena safena parva. Rev. U.D.C.A Act. \& Div. Cient. 17(2): 325-331. 